\title{
POSITIVE SOLUTIONS FOR ASYMPTOTICALLY LINEAR ELLIPTIC SYSTEMS
}

\author{
CHAOQUAN PENG \\ Wuhan Institute of Physics and Mathematics, Chinese Academy of Sciences, P.O.Box 71010, Wuhan 430071, \\ and Graduate School, Chinese Academy of Sciences, Beijing 100049, The People's Republic of China \\ e-mail:pcq1979@163.com \\ and JIANFU YANG \\ Department of Mathematics, Jiangxi Normal University, Nanchang, Jiangxi 330022, P.R. China \\ e-mail: jfyang_2000@yahoo.com
}

(Received 3 November, 2006; revised 2 February, 2007; accepted 13 February, 2007)

Abstract. In this paper, we show that the semilinear elliptic systems of the form

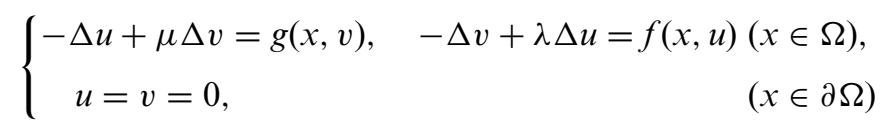

possess at least one positive solution pair $(u, v) \in H_{0}^{1}(\Omega) \times H_{0}^{1}(\Omega)$, where $\Omega$ is a smooth bounded domain in $\mathbb{R}^{N}, f(x, t)$ and $g(x, t)$ are continuous functions on $\Omega \times \mathbb{R}$ and asymptotically linear at infinity.

2000 Mathematics Subject Classification. 35J60, 35J65.

1. Introduction. In this paper, we consider the existence of positive solutions of nonlinear elliptic systems

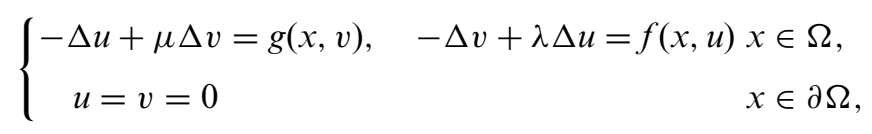

where $\Omega \subset \mathbb{R}^{N}$ is a smooth bounded domain, $\lambda$ and $\mu$ are nonnegative numbers, $f(x, t)$ and $g(x, t)$ are functions continuous on $\Omega \times \mathbb{R}$ and asymptotically linear at infinity for $t$.

In the case of $\lambda=\mu=0$, there is much research in the literature for the case in which $f$ and $g$ are superlinear. See [1], [2], [3], [5], [13] and references therein. In [8] $\mathrm{G}$. Li and the second author considered the asymptotically linear elliptic systems

$$
-\Delta u+u=g(x, v), \quad-\Delta v+v=f(x, u) \quad\left(x \in \mathbb{R}^{N}\right)
$$

and obtained a positive solution by using the linking theorem under the Cerami compactness condition.

If $\lambda, \mu \neq 0$, the problem has some new features. First, by the Pohozaev type identity, the parameters $\lambda$ and $\mu$ affect the subcritical range of the growth of nonlinear terms at infinity. See [10]. Secondly, the decomposition of the space in the framework 
involves the parameters. In fact, let $E=H_{0}^{1}(\Omega) \times H_{0}^{1}(\Omega)$ be equipped with the norm

$$
\|z\|_{E}=\left(\int_{\Omega}\left(|\nabla u|^{2}+|\nabla v|^{2}\right) d x\right)^{\frac{1}{2}},
$$

where $z=(u, v)$. We define a bilinear form $B: E \times E \rightarrow \mathbb{R}$ by

$$
B[(u, v),(\varphi, \psi)]=\int_{\Omega}(\nabla u \nabla \psi-\lambda \nabla u \nabla \varphi+\nabla v \nabla \varphi-\mu \nabla v \nabla \psi) d x .
$$

Then $B[z, \eta]=B[\eta, z], \forall z, \eta \in E$. Note that $B$ induces a self-adjoint bounded linear operator $L: E \rightarrow E$ such that

$$
B[z, \eta]=\langle L z, \eta\rangle_{E} \quad(\forall z, \eta \in E) .
$$

The eigenvalue problem

$$
L z=k z
$$

has two eigenvalues

$$
k_{ \pm}=\frac{-\lambda-\mu \pm \sqrt{(\lambda-\mu)^{2}+4}}{2} .
$$

The corresponding eigenvectors are $\left(u,\left(k_{ \pm}+\lambda\right) u\right)$, where $u \in H_{0}^{1}(\Omega)$. Let

$$
E^{ \pm}=\left\{\left(u, \frac{\lambda-\mu \pm \sqrt{(\lambda-\mu)^{2}+4}}{2} u\right), \text { where } u \in H_{0}^{1}(\Omega)\right\} .
$$

Then $E=E^{+} \oplus E^{-}$. Also, both $E^{+}$and $E^{-}$are infinite dimensional. We may write for $z=(u, v) \in E$,

$$
z^{+}=\frac{1}{k_{2}-k_{1}}\left(k_{2} u-v,-u-k_{1} v\right), \quad z^{-}=\frac{1}{k_{2}-k_{1}}\left(-k_{1} u+v, u+k_{2} v\right),
$$

and we have

$$
B\left[z^{+}, z^{-}\right]=\left\langle L z^{+}, z^{-}\right\rangle_{E}=0, \quad \forall z^{ \pm} \in E^{ \pm} .
$$

We may verify that

$$
\langle z, \eta\rangle=B\left[z^{+}-z^{-}, \eta\right], \quad \forall z, \eta \in E
$$

is an inner product in $E$ that induces a norm $\|z\|=(\langle z, z\rangle)^{\frac{1}{2}}, z \in E$. The subspaces $E^{+}$ and $E^{-}$are orthogonal with respect to the inner product $\langle\cdot, \cdot\rangle$. Moreover, we have

$$
\|z\|^{2}=\left\|z^{+}\right\|^{2}+\left\|z^{-}\right\|^{2}=B\left[z^{+}-z^{-}, z^{+}+z^{-}\right] .
$$

Let

$$
k_{1}=\frac{\lambda-\mu+\sqrt{(\lambda-\mu)^{2}+4}}{2}, \quad k_{2}=\frac{\lambda-\mu-\sqrt{(\lambda-\mu)^{2}+4}}{2} .
$$

Note that

$$
-\mu k_{1}^{2}+2 k_{1}-\lambda>0 \text { if and only if } \lambda \mu<1
$$


and $k_{1} k_{2}=-1$. We know that $E^{+}$and $E^{-}$are also orthogonal with respect to the inner product $\langle\cdot, \cdot\rangle_{E}$. The norms $\|\cdot\|$ and $\|\cdot\|_{E}$ are then equivalent if $\lambda \mu<1$.

In [10], the authors considered problem (1.1) with superlinear nonlinearities. In this paper, we consider the case in which $f(x, t)$ and $g(x, t)$ are asymptotically linear at infinity for $t$.

We assume that $f$ and $g$ satisfy the following conditions.

$(H 1) f, g \in C^{1}(\Omega \times \mathbb{R}, \mathbb{R})$.

(H2) $\lim _{t \rightarrow 0}(f(x, t) / t)=\lim _{t \rightarrow 0}(g(x, t) / t)=0$ uniformly with respect to $x \in \Omega$ and $f(x, t)>0, g(x, t)>0$ for $t>0, x \in \Omega$.

(H3) $\lim _{t \rightarrow \infty}(f(x, t) / t)=l>0, \lim _{t \rightarrow \infty}(g(x, t) / t)=m>0$ uniformly in $x \in \Omega$.

(H4) $f(x, t) / t$ and $g(x, t) / t$ are non-decreasing in $t \geq 0$ for $x \in \Omega$.

(H5) $\frac{1}{2} t f(x, t)-F(x, t)>0$ and $\frac{1}{2} \operatorname{tg}(x, t)-G(x, t)>0$ for any $(x, t) \in \Omega \times \mathbb{R}^{+}$. Also there are $\delta \in(0,1)$ and $c_{\delta}>0$ such that $f(x, t) / t \geq \delta$ and $g(x, t) / t \geq \delta$ imply, respectively, that

$$
\frac{1}{2} t f(x, t)-F(x, t) \geq c_{\delta}, \quad \frac{1}{2} \operatorname{tg}(x, t)-G(x, t) \geq c_{\delta},
$$

where $F(x, t)=\int_{0}^{t} f(x, s) d s, G(x, t)=\int_{0}^{t} g(x, s) d s$.

Let $\lambda_{1}$ be the first eigenvalue of $\left(-\Delta, H_{0}^{1}(\Omega)\right)$ and $\varphi_{1}>0$ be a corresponding eigenfunction.

The main result of this paper is the following theorem.

THeorem 1.1. Suppose that $(H 1)-(H 5)$. If $0 \leq \lambda \mu<1$ and $\lambda_{1}<$ $\frac{m \lambda+\mu l+\sqrt{(m \lambda-\mu l)^{2}+4 m l}}{2(1-\lambda \mu)}$, then the problem (1.1) possesses at least one positive solution pair $z=(u, v) \in E$. Suppose further $0 \leq \lambda+\mu<2$. Then the problem (1.1) possesses a least energy positive solution pair $z=(u, v) \in E$.

Theorem 1.1 will be proved by looking for critical points of the associated functional

$$
I(u, v)=\int_{\Omega}\left(\nabla u \nabla v-\frac{\lambda}{2}|\nabla u|^{2}-\frac{\mu}{2}|\nabla v|^{2}\right) d x-\int_{\Omega} F(x, u) d x-\int_{\Omega} G(x, v) d x
$$

defined on $E=H_{0}^{1}(\Omega) \times H_{0}^{1}(\Omega)$. In order to find critical points of $I$, we show first that the functional $I$ has a geometry of linking type. Then, we find a $(P S)_{c}$ sequence $\left\{z_{n}\right\} \subset E$ of $I$ by a linking type theorem in [6]. Theorem 1.1 follows by showing $\left\{z_{n}\right\}$ has a strongly convergent subsequence. As a byproduct, we show that

$$
I^{\infty}=\inf \left\{I(z): I^{\prime}(z)=0, z=(u, v) \in E \backslash\{0\}\right\}
$$

is achieved by some $z_{0}=\left(u_{0}, v_{0}\right)$ with $u_{0}>0, v_{0}>0$.

In the same way, we consider the problem

$$
\left\{\begin{array}{cl}
-\Delta u-\mu \Delta v=g(x, v), \quad-\Delta v+\lambda \Delta u=f(x, u) & (x \in \Omega), \\
u=v=0 & (x \in \partial \Omega),
\end{array}\right.
$$


and

$$
\left\{\begin{array}{cl}
-\Delta u+\mu \Delta v=g(x, v), \quad-\Delta v-\lambda \Delta u=f(x, u) & (x \in \Omega), \\
u=v=0 & (x \in \partial \Omega) .
\end{array}\right.
$$

We have the following results.

THEOREM 1.2. Suppose that $(H 1)-(H 5)$ hold. If $\lambda, \mu \geq 0$ and $\lambda_{1}<$ $\frac{m \lambda-\mu l+\sqrt{(m \lambda+\mu l)^{2}+4 m l}}{2(1+\lambda \mu)}$, then the problem (1.8) possesses at least one nonnegative nontrivial solution pair $z=(u, v) \in E$.

THEOREM 1.3. Suppose that $(H 1)-(H 5)$ hold. If $\lambda, \mu \geq 0$ and $\lambda_{1}<$ $\frac{\mu l-m \lambda+\sqrt{(m \lambda+\mu l)^{2}+4 m l}}{2(1+\lambda \mu)}$, then the problem (1.9) possesses at least one nonnegative nontrivial solution pair $z=(u, v) \in E$.

Theorem 1.1 will be proved in Section 2.

2. Existence results. Suppose in this section that $\lambda, \mu$ satisfies $\lambda \mu<1$. This allows us to define an equivalent norm on $E$. As we are only interested in positive solutions, we assume in the following that $f(x, t)=g(x, t)=0$ if $t \leq 0$. It is known that the energy functional $I$ defined in (1.7) is $C^{1}$ on $E$ with the Fréchet derivative $I^{\prime}$ satisfying

$$
\begin{aligned}
\left\langle I^{\prime}(u, v),(\varphi, \psi)\right\rangle= & \int_{\Omega}[\nabla u \nabla \psi+\nabla v \nabla \varphi-\lambda \nabla u \nabla \varphi-\mu \nabla v \nabla \psi] d x-\int_{\Omega} f(x, u) \varphi d x \\
& -\int_{\Omega} g(x, v) \psi d x
\end{aligned}
$$

for $(u, v),(\varphi, \psi) \in E$.

A sequence $\left\{z_{n}\right\} \subset E$ is called a Palais-Smale sequence of a $C^{1}$ functional I on $E$ at level $c\left((P S)_{c}\right.$-sequence for short) if $I\left(z_{n}\right) \rightarrow c$ and $I^{\prime}\left(z_{n}\right) \rightarrow 0$ as $n \rightarrow \infty$. To get a $(P S)_{c}$-sequence, we use the linking theorem in [6].

Proposition 2.1. (Theorem 3.4 of [6])

Let $E$ be a real Hilbert space and suppose that $\Phi \in C^{1}(E, \mathbb{R})$ satisfies the following hypotheses:

(i) $\Phi(u)=\frac{1}{2}\langle L u, u\rangle-\Psi(u)$, where $L$ is a bounded self-adjoint linear operator, $\Psi$ is bounded below, weakly sequentially lower semicontinuous and $\nabla \Psi$ is weakly sequentially continuous;

(ii) there exists a closed separable L-invariant subspace $Y$ such that the quadratic form $u \mapsto\langle L u, u\rangle$ is negative definite on $Y$ and positive semi-definite on $Y^{\perp}$;

(iii) there are constants $b, \rho>0$ such that $\left.\Phi\right|_{S_{\rho} \cap Y^{\perp}} \geq b$;

(iv) there is $z_{0} \in S_{1} \cap Y^{\perp}$ and $R>\rho$ such that $\left.\Phi\right|_{\partial M} \leq 0$, where $M:=\{u=y+$ $\left.\lambda z_{0}: y \in Y,\|u\|<R, \lambda>0\right\}$.

Then there exists a sequence $\left\{u_{n}\right\} \subset E$ such that $\nabla \Phi\left(u_{n}\right) \rightarrow 0$ and $\Phi\left(u_{n}\right) \rightarrow c$ for some $c \in\left[b, \sup _{\bar{M}} \Phi\right]$.

For $z_{0} \in E^{+} \backslash\{0\}$ and $R>r>0$, let

$$
M_{R}=\left\{z=z^{-}+\rho z_{0}: z^{-} \in E^{-}, \rho \geq 0,\|z\| \leq R\right\}, \quad N_{r}=\left\{z \in E^{+}:\|z\|=r\right\} .
$$

Let $\Psi(u, v)=\int_{\Omega} F(x, u) d x+\int_{\Omega} G(x, v) d x$. 
LEMMA 2.1. The following properties hold.

(i) There exist $r, \alpha>0$ and $R>r$ ( $R$ depending on $z_{0}$ ) such that $I(z) \geq \alpha$ for all $z \in N_{r}$ and $I(z) \leq 0$ for all $z \in \partial M_{R}$.

(ii) $\Psi \geq 0, \Psi$ is weakly sequentially lower semicontinuous and $\Psi^{\prime}$ is weakly sequentially continuous.

Proof. We first prove (i). For $z \in E^{+}$, there is a $u \in H_{0}^{1}(\Omega)$ such that $z=\left(u, k_{1} u\right)$. By $\left(H_{1}\right)-\left(H_{3}\right)$, we have for $\epsilon>0$ that

$$
I(z) \geq \frac{1}{2}\|z\|^{2}-\int_{\Omega}\left(c \epsilon u^{2}+c_{\epsilon} u^{p}\right) d x \geq \frac{1}{4}\|z\|^{2}-C\|z\|^{p},
$$

where $p \in\left(2,2^{*}\right), 2^{*}=\frac{2 N}{N-2}$. Hence for $\|z\|=r$ sufficiently small, there is $\alpha>0$ such that

$$
b:=\inf _{N_{r}} I \geq \alpha
$$

Let $z_{0}=\left(u_{0}, k_{1} u_{0}\right) \in E^{+} \backslash\{0\}$ with $\left\|z_{0}\right\|=1$. Note that $u_{0}$ will be specified later, such that

$$
1-\min (l, m) \int_{\Omega} u_{0}^{2} d x<0 .
$$

We prove now that there exists a $R>r$ such that $\max _{\partial M_{R}} I=0$. Set

$$
u_{0}=\left(\left(-\mu k_{1}^{2}+2 k_{1}-\lambda\right) \beta D(N)(d(N))^{2}\right)^{-\frac{1}{2}} \beta^{\frac{N}{4}} e^{-\beta|x|^{2}},
$$

where

$$
(d(N))^{2}=\int_{\Omega} e^{-2|x|^{2}} d x, \quad D(N)=4(d(N))^{-2} \int_{\Omega}|x|^{2} e^{-2|x|^{2}} d x
$$

and $\beta$ will be determined later. Then we have as [9] that

$$
\int_{\Omega} u_{0}^{2} d x=\frac{1}{\left(-\mu k_{1}^{2}+2 k_{1}-\lambda\right) \beta D(N)} \quad \text { and } \quad \int_{\Omega}\left|\nabla u_{0}\right|^{2} d x=\frac{1}{\left(-\mu k_{1}^{2}+2 k_{1}-\lambda\right)}
$$

which yields $\left\|z_{0}\right\|=1$. Choosing $\beta \in\left(0, \min (l, m) /\left(\mu k_{1}^{2}+2 k_{1}-\lambda\right) D(N)\right)$, we obtain

$$
1-\min (l, m) \int_{\Omega} u_{0}^{2} d x<0 .
$$

If $z \in \partial M_{R}$, then $z=z^{-}+s z_{0}$ with either $\|z\|=R$, for $s \geq 0$, or $\|z\|<R$, when $s=0$. If $s=0$, we have $z \in E^{-}, z=\left(u, k_{2} u\right)$ and

$$
I\left(u, k_{2} u\right)=-\frac{1}{2}\left\|z^{-}\right\|^{2}-\int_{\Omega} F(x, u) d x-\int_{\Omega} G\left(x, k_{2} u\right) d x \leq 0,
$$

because $F(x, t), G(x, t) \geq 0$ for any $(x, t) \in \mathbb{R}^{N} \times \mathbb{R}$.

Suppose now that $s>0$. If the conclusion were not true, we would have a sequence $\left\{z_{n}\right\} \subset \partial M_{n}, z_{n}=s_{n} z_{0}+z_{n}^{-}, s_{n}>0,\left\|z_{n}\right\|=n$ such that $I\left(z_{n}\right)>0$. That is, 
if $z_{n}=\left(u_{n}, v_{n}\right):=\left(s_{n} u_{0}+\varphi_{n}, s_{n} k_{1} u_{0}+k_{2} \varphi_{n}\right)$, then

$$
I\left(z_{n}\right)=\frac{1}{2}\left(s_{n}^{2}\left\|z_{0}\right\|^{2}-\left\|z_{n}^{-}\right\|^{2}\right)-\int_{\Omega} F\left(x, u_{n}\right) d x-\int_{\Omega} G\left(x, v_{n}\right) d x>0 .
$$

Hence

$$
\frac{I\left(z_{n}\right)}{\left\|z_{n}\right\|^{2}}=\frac{1}{2}\left(\frac{s_{n}^{2}}{\left\|z_{n}\right\|^{2}}\left\|z_{0}\right\|^{2}-\frac{\left\|z_{n}^{-}\right\|^{2}}{\left\|z_{n}\right\|^{2}}\right)-\int_{\Omega} \frac{F\left(x, u_{n}\right)+G\left(x, v_{n}\right)}{\left\|z_{n}\right\|^{2}} d x>0 .
$$

As a result, $s_{n} \geq\left\|z_{n}^{-}\right\|$. On the other hand,

$$
\frac{s_{n}^{2}\left\|z_{0}\right\|^{2}+\left\|z_{n}^{-}\right\|^{2}}{\left\|z_{n}\right\|^{2}}=1
$$

implies that

$$
\frac{s_{n}^{2}}{\left\|z_{n}\right\|^{2}} \rightarrow \rho^{2} \geq 0
$$

for some $\rho \geq 0$, and

$$
\zeta_{n}^{-}:=\frac{z_{n}^{-}}{\left\|z_{n}\right\|} \rightarrow \zeta^{-}=\left(\varphi, k_{2} \varphi\right) \in E
$$

as $n \rightarrow \infty$.

If $\rho=0$, we get from (2.2) that

$$
\frac{\left\|z_{n}^{-}\right\|^{2}}{\left\|z_{n}\right\|^{2}} \rightarrow 0, \quad \int_{\Omega} \frac{F\left(x, u_{n}\right)}{\left\|z_{n}\right\|^{2}} d x \rightarrow 0, \quad \int_{\Omega} \frac{G\left(x, v_{n}\right)}{\left\|z_{n}\right\|^{2}} d x \rightarrow 0
$$

as $n \rightarrow \infty$. Therefore,

$$
1=\frac{s_{n}^{2}}{\left\|z_{n}\right\|^{2}}\left\|z_{0}\right\|^{2}+\frac{\left\|z_{n}^{-}\right\|^{2}}{\left\|z_{n}\right\|^{2}} \rightarrow 0
$$

as $n \rightarrow \infty$, which is impossible.

If $\rho>0$, since $s_{n}^{2} /\left\|z_{n}\right\|^{2} \rightarrow \rho^{2}>0$ and $\left\|z_{n}\right\| \rightarrow+\infty$ as $n \rightarrow \infty$, it follows that $s_{n} \rightarrow+\infty$ as $n \rightarrow \infty$. If $x \in \Omega$ is such that $\rho u_{0}(x)+\varphi(x) \neq 0$, we have

$$
\lim _{n \rightarrow \infty} \frac{s_{n} u_{0}(x)+\varphi_{n}(x)}{\left\|z_{n}\right\|}=\rho u_{0}(x)+\varphi(x) \neq 0 ;
$$

thus, $u_{n}=s_{n} u_{0}(x)+\varphi_{n}(x) \rightarrow \infty$ as $n \rightarrow \infty$. Similarly, if $\rho k_{1} u_{0}(x)+k_{2} \varphi(x) \neq$ 0 , we have $v_{n}=s_{n} k_{1} u_{0}(x)+k_{2} \varphi_{n}(x) \rightarrow \infty$ as $n \rightarrow \infty$. As $I\left(z_{n}\right) /\left\|z_{n}\right\|^{2}>0$ and $F(x, t), G(x, t) \geq 0$, we deduce that

$$
\begin{aligned}
0< & \frac{s_{n}^{2}}{2\left\|z_{n}\right\|^{2}}\left\|z_{0}\right\|^{2}-\frac{\left\|z_{n}^{-}\right\|^{2}}{2\left\|z_{n}\right\|^{2}}-\int_{\Omega}\left[\frac{F\left(x, u_{n}\right)}{u_{n}^{2}}\left(\frac{u_{n}}{\left\|z_{n}\right\|}\right)^{2}+\frac{G\left(x, v_{n}\right)}{v_{n}^{2}}\left(\frac{v_{n}}{\left\|z_{n}\right\|}\right)^{2}\right] d x \\
\leq & \frac{s_{n}^{2}}{2\left\|z_{n}\right\|^{2}}\left\|z_{0}\right\|^{2}-\frac{\left\|z_{n}^{-}\right\|^{2}}{2\left\|z_{n}\right\|^{2}}-\int_{\left\{\rho u_{0}+\varphi \neq 0\right\}} \frac{F\left(x, u_{n}\right)}{u_{n}^{2}}\left(\frac{u_{n}}{\left\|z_{n}\right\|}\right)^{2} d x \\
& -\int_{\left\{\rho k_{1} u_{0}+k_{2} \varphi \neq 0\right\}} \frac{G\left(x, v_{n}\right)}{v_{n}^{2}}\left(\frac{v_{n}}{\left\|z_{n}\right\|}\right)^{2} d x .
\end{aligned}
$$


Notice that

$$
\frac{u_{n}}{\left\|z_{n}\right\|}=\frac{s_{n} u_{0}+\varphi_{n}}{\left\|z_{n}\right\|} \rightarrow \rho u_{0}+\varphi \quad \text { and } \quad \frac{v_{n}}{\left\|z_{n}\right\|}=\frac{s_{n} k_{1} u_{0}+k_{2} \varphi_{n}}{\left\|z_{n}\right\|} \rightarrow \rho k_{1} u_{0}+k_{2} \varphi
$$

in $H_{0}^{1}(\Omega)$ as $n \rightarrow \infty$. By Sobolev's is embedding theorems, we may assume that

$$
\frac{u_{n}}{\left\|z_{n}\right\|}=\frac{s_{n} u_{0}+\varphi_{n}}{\left\|z_{n}\right\|} \rightarrow \rho u_{0}+\varphi, \quad \frac{v_{n}}{\left\|z_{n}\right\|}=\frac{s_{n} k_{1} u_{0}+k_{2} \varphi_{n}}{\left\|z_{n}\right\|} \rightarrow \rho k_{1} u_{0}+k_{2} \varphi \text { a.e. in } \Omega
$$

as $n \rightarrow \infty$. Let $z=\rho z_{0}+\zeta^{-}$and $z_{0}=\left(u_{0}, k_{1} u_{0}\right), \zeta^{-}=\left(\varphi, k_{2} \varphi\right)$. Taking the limit in (2.3), using Fatou's Lemma and the fact that $\liminf _{n \rightarrow \infty}\left(\left\|z_{n}^{-}\right\| /\left\|z_{n}\right\|\right) \geq\left\|\zeta^{-}\right\|$, we obtain by (2.1) that

$$
\begin{aligned}
0 & \leq \frac{1}{2}\left(\rho^{2}\left\|z_{0}\right\|^{2}-\left\|\zeta^{-}\right\|^{2}\right)-\frac{l}{2} \int_{\left\{\rho u_{0}+\varphi \neq 0\right\}}\left(\rho u_{0}+\varphi\right)^{2} d x-\frac{m}{2} \int_{\left\{\rho k_{1} u_{0}+k_{2} \varphi \neq 0\right\}}\left(\rho k_{1} u_{0}+k_{2} \varphi\right)^{2} d x \\
& =\frac{1}{2}\left(\rho^{2}\left\|z_{0}\right\|^{2}-\left\|\zeta^{-}\right\|^{2}\right)-\frac{1}{2} \int_{\Omega}\left[l\left(\rho u_{0}+\varphi\right)^{2}+m\left(\rho k_{1} u_{0}+k_{2} \varphi\right)^{2}\right] d x \\
& \leq \frac{1}{2}\left(\rho^{2}\left\|z_{0}\right\|^{2}-\left\|\zeta^{-}\right\|^{2}\right)-\frac{1}{2} \min (l, m) \int_{\Omega}\left[\left(\rho u_{0}+\varphi\right)^{2}+\left(\rho k_{1} u_{0}+k_{2} \varphi\right)^{2}\right] d x \\
& =\frac{1}{2}\left(\rho^{2}\left\|z_{0}\right\|^{2}-\left\|\zeta^{-}\right\|^{2}\right)-\frac{1}{2} \min (l, m) \int_{\Omega}\left[\rho^{2} u_{0}^{2}\left(1+k_{1}^{2}\right)+\varphi^{2}\left(1+k_{2}^{2}\right)\right] d x \\
& \leq \frac{1}{2}\left(\rho^{2}-\left\|\zeta^{-}\right\|^{2}\right)-\frac{1}{2} \min (l, m) \int_{\Omega}\left[\rho^{2} u_{0}^{2}+\varphi^{2}\right] d x \\
& =\frac{1}{2} \rho^{2}\left[1-\min (l, m) \int_{\Omega} u_{0}^{2} d x\right]-\frac{1}{2}\left\|\zeta^{-}\right\|^{2}-\frac{1}{2} \min (l, m) \int_{\Omega} \varphi^{2} d x \\
& <0
\end{aligned}
$$

which is a contradiction. Hence, part (i) of the Lemma is proved.

Now we prove (ii). It is obvious that $\Psi \geq 0$. Let $z_{n}=\left(u_{n}, v_{n}\right) \rightarrow z=(u, v)$ in $E$ as $n \rightarrow \infty$. Then $u_{n} \rightarrow u, v_{n} \rightarrow v$ in $L^{p}(\Omega), p \in\left(2,2^{*}\right)$, and $u_{n} \rightarrow u, v_{n} \rightarrow v$ a.e. in $\Omega$ possibly after passing to a subsequence as $n \rightarrow \infty$. It follows from Fatou's Lemma that $\Psi$ is weakly sequentially lower semicontinuous. Moreover, by $\left(H_{1}\right)-\left(H_{3}\right)$, there is a $2<p<2^{*}$ if $N \geq 3$ and $2<p<+\infty$ if $N \leq 2$, such that for $\epsilon>0$ we can find $c_{\epsilon}>0$ such that

$$
|f(x, t)| \leq \epsilon|t|+c_{\epsilon}|t|^{p-1}, \quad|g(x, t)| \leq \epsilon|t|+c_{\epsilon}|t|^{p-1},
$$

for $(x, t) \in \Omega \times \mathbb{R}$. Therefore,

$$
\int_{\Omega}\left[f\left(x, u_{n}\right)-f(x, u)\right] \varphi d x=o(1), \quad \int_{\Omega}\left[g\left(x, v_{n}\right)-g(x, v)\right] \psi d x=o(1)
$$

as $n \rightarrow \infty$. We deduce from

$$
\left\langle\Psi^{\prime}\left(z_{n}\right)-\Psi^{\prime}(z),(\varphi, \psi)\right\rangle=\int_{\Omega}\left[f\left(x, u_{n}\right)-f(x, u)\right] \varphi d x+\int_{\Omega}\left[g\left(x, v_{n}\right)-g(x, v)\right] \psi d x
$$


where $(\varphi, \psi) \in E$, that $\Psi^{\prime}$ is weakly sequentially continuous. The Lemma is proved.

PROPOSITION 2.2. If $(u, v) \in H_{0}^{1}(\Omega) \times H_{0}^{1}(\Omega)$ is a positive solution of (1.1), then $\lambda_{1} \leq \frac{m \lambda+\mu l+\sqrt{(m \lambda-\mu l)^{2}+4 m l}}{2(1-\lambda \mu)}$.

Proof. Let $k=\frac{m \lambda-\mu l+\sqrt{(m \lambda-\mu l)^{2}+4 m l}}{2 m}$. It is apparent that $(u, v)=(u, k \tilde{v})$ is a positive solution pair of the problem

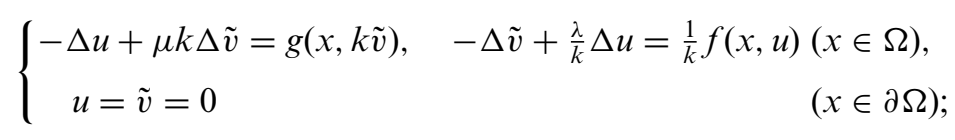

that is

$$
-\left(1-\frac{\lambda}{k}\right) \Delta\left(u+\frac{1-\mu k}{1-\frac{\lambda}{k}} \tilde{v}\right)=g(x, k \tilde{v})+\frac{1}{k} f(x, u) .
$$

By $\left(H_{3}\right)$ and $\left(H_{4}\right)$, we have

$$
\begin{aligned}
\left(1-\frac{\lambda}{k}\right) \int_{\Omega}\left|\nabla\left(u+\frac{1-\mu k}{1-\frac{\lambda}{k}} \tilde{v}\right)\right|^{2} d x & =\int_{\Omega}\left[g(x, k \tilde{v})+\frac{1}{k} f(x, u)\right]\left(u+\frac{1-\mu k}{1-\frac{\lambda}{k}} \tilde{v}\right) d x \\
& \leq \int_{\Omega}\left[m k \tilde{v}+\frac{l}{k} u\right]\left(u+\frac{1-\mu k}{1-\frac{\lambda}{k}} \tilde{v}\right) d x \\
& =\frac{l}{k} \int_{\Omega}\left(u+\frac{m k^{2}}{l} \tilde{v}\right)\left(u+\frac{1-\mu k}{1-\frac{\lambda}{k}} \tilde{v}\right) d x .
\end{aligned}
$$

By the definition of $k$ we know that $\frac{1-\mu k}{1-\frac{\lambda}{k}}=\frac{m k^{2}}{l}$, and then

$$
\lambda_{1} \leq \frac{\frac{l}{k}}{1-\frac{\lambda}{k}}=\frac{l}{k-\lambda}=\frac{m \lambda+\mu l+\sqrt{(m \lambda-\mu l)^{2}+4 m l}}{2(1-\lambda \mu)} .
$$

The proof is complete.

LEMMA 2.2. Let $\left\{z_{n}\right\}$ be a $(P S)_{c}$-sequence of I. If $\lambda_{1}<\frac{m \lambda+\mu l+\sqrt{(m \lambda-\mu l)^{2}+4 m l}}{2(1-\lambda \mu)}$, then $\left\{z_{n}\right\}$ is relatively compact in $E$.

Proof. It is sufficient to show that $\left\{z_{n}\right\}$ is bounded in $E$. Suppose, to the contrary, that $\left\|z_{n}\right\| \rightarrow \infty$ as $n \rightarrow \infty$. Let

$$
w_{n}=\frac{z_{n}}{\left\|z_{n}\right\|}=\left(\frac{u_{n}}{\left\|z_{n}\right\|}, \frac{v_{n}}{\left\|z_{n}\right\|}\right) \triangleq\left(w_{n}^{1}, w_{n}^{2}\right), \quad \rho_{n}(x)=\left|w_{n}(x)\right|^{2}=\frac{1}{\left\|z_{n}\right\|^{2}}\left(u_{n}^{2}+v_{n}^{2}\right) .
$$

Then we may assume for some $w=\left(w_{1}, w_{2}\right) \in E$ that

$$
w_{n}=\left(w_{n}^{1}, w_{n}^{2}\right) \rightarrow w=\left(w_{1}, w_{2}\right) \in E,
$$

and

$$
w_{n}=\left(w_{n}^{1}, w_{n}^{2}\right) \rightarrow w=\left(w_{1}, w_{2}\right) \text { a.e. in } \Omega
$$


as $n \rightarrow \infty$. If $w=\left(w_{1}, w_{2}\right)=0$, then by the Sobolev embedding theorem, we would have

$$
w_{n}^{1} \rightarrow 0, w_{n}^{2} \rightarrow 0 \text { in } L^{q}(\Omega)
$$

as $n \rightarrow \infty$ for $2 \leq q<2 N /(N-2)$. Since

$z_{n}^{+}=\frac{1}{k_{2}-k_{1}}\left(\left(k_{2} u_{n}-v_{n}\right),\left(-u_{n}-k_{1} v_{n}\right)\right), \quad z_{n}^{-}=\frac{1}{k_{2}-k_{1}}\left(\left(-k_{1} u_{n}+v_{n}\right),\left(u_{n}+k_{2} v_{n}\right)\right)$,

we have

$$
u_{n}^{+}=\frac{k_{2} u_{n}-v_{n}}{k_{2}-k_{1}}, \quad u_{n}^{-}=\frac{-u_{n}-k_{1} v_{n}}{k_{2}-k_{1}}
$$

and

$$
v_{n}^{+}=\frac{-k_{1} u_{n}+v_{n}}{k_{2}-k_{1}}, \quad v_{n}^{-}=\frac{u_{n}+k_{2} v_{n}}{k_{2}-k_{1}}
$$

Thus,

$$
\left\langle I^{\prime}\left(z_{n}\right), z_{n}^{+}\right\rangle=\left\|z_{n}^{+}\right\|^{2}-\int_{\Omega} f\left(x, u_{n}\right) u_{n}^{+} d x-\int_{\Omega} g\left(x, v_{n}\right) v_{n}^{+} d x=o(1)\left\|z_{n}^{+}\right\|
$$

and

$$
\left\langle I^{\prime}\left(z_{n}\right), z_{n}^{-}\right\rangle=-\left\|z_{n}^{-}\right\|^{2}-\int_{\Omega} f\left(x, u_{n}\right) u_{n}^{-} d x-\int_{\Omega} g\left(x, v_{n}\right) v_{n}^{-} d x=o(1)\left\|z_{n}^{-}\right\|,
$$

where $z_{n}^{ \pm}=\left(u_{n}^{ \pm}, v_{n}^{ \pm}\right)$. This yields

$$
\left\|z_{n}\right\|^{2}-\int_{\Omega} f\left(x, u_{n}\right)\left(u_{n}^{+}-u_{n}^{-}\right) d x-\int_{\Omega} g\left(x, v_{n}\right)\left(v_{n}^{+}-v_{n}^{-}\right) d x=o(1)\left(\left\|z_{n}^{+}\right\|-\left\|z_{n}^{-}\right\|\right),
$$

and so

$$
\int_{\Omega} \frac{f\left(x, u_{n}\right)\left(u_{n}^{+}-u_{n}^{-}\right)+g\left(x, v_{n}\right)\left(v_{n}^{+}-v_{n}^{-}\right)}{\left\|z_{n}\right\|^{2}} d x=o(1)+1 .
$$

For $\delta$ and $c_{\delta}$ given in $\left(H_{5}\right)$, let

$$
A_{n}=\left\{x \in \Omega:\left|f\left(x, u_{n}(x)\right)\right| \leq \delta\left|u_{n}(x)\right|\right\}, \quad B_{n}=\left\{x \in \Omega:\left|g\left(x, u_{n}(x)\right)\right| \leq \delta\left|u_{n}(x)\right|\right\},
$$

and $\Omega_{n}=A_{n} \cap B_{n}$. We deduce that

$$
\begin{aligned}
& \left|\int_{\Omega_{n}} \frac{f\left(x, u_{n}\right)\left(u_{n}^{+}-u_{n}^{-}\right)+g\left(x, v_{n}\right)\left(v_{n}^{+}-v_{n}^{-}\right)}{\left\|z_{n}\right\|^{2}} d x\right| \\
\leq & \int_{\Omega_{n}} \frac{\left|f\left(x, u_{n}\right)\left(u_{n}^{+}-u_{n}^{-}\right)+g\left(x, v_{n}\right)\left(v_{n}^{+}-v_{n}^{-}\right)\right|}{\left\|z_{n}\right\|^{2}} d x \\
\leq & c \delta \int_{\Omega_{n}} \frac{\left|u_{n}\right|^{2}+\left|v_{n}\right|^{2}}{\left\|z_{n}\right\|^{2}} d x \leq c \delta \int_{\Omega_{n}}\left|w_{n}\right|^{2} d x \\
\leq & \delta<1
\end{aligned}
$$


for $n$ large enough. Thus, we have for all $n$ sufficiently large

$$
\int_{\Omega \backslash \Omega_{n}} \frac{f\left(x, u_{n}\right)\left(u_{n}^{+}-u_{n}^{-}\right)+g\left(x, v_{n}\right)\left(v_{n}^{+}-v_{n}^{-}\right)}{\left\|z_{n}\right\|^{2}} d x \geq 1-\delta+o(1) .
$$

On the other hand, $\left(\mathrm{H}_{2}\right)$ and $\left(\mathrm{H}_{3}\right)$ imply that there exists $c>0$ such that

$$
|f(x, t)|, \quad|g(x, t)| \leq c+c|t|
$$

for $(x, t) \in \Omega \times \mathbb{R}$. By (2.5) and (2.6), we have

$$
\begin{aligned}
0<1-\delta+o(1) & \leq \int_{\Omega \backslash \Omega_{n}} \frac{f\left(x, u_{n}\right)\left(u_{n}^{+}-u_{n}^{-}\right)+g\left(x, v_{n}\right)\left(v_{n}^{+}-v_{n}^{-}\right)}{\left\|z_{n}\right\|^{2}} d x \\
& \leq c \int_{\Omega \backslash \Omega_{n}} \frac{\left|u_{n}\right|^{2}+\left|v_{n}\right|^{2}}{\left\|z_{n}\right\|^{2}} d x \leq c \int_{\Omega \backslash \Omega_{n}}\left|w_{n}\right|^{2} d x \\
& \leq \operatorname{mes}\left\{\Omega \backslash \Omega_{n}\right\}^{\frac{p-2}{p}}\left\|w_{n}\right\|_{L^{p}(\Omega)}^{2} \rightarrow 0
\end{aligned}
$$

as $n \rightarrow \infty$ because $\left\|w_{n}\right\|_{L^{q}(\Omega)} \rightarrow 0$ for $2 \leq q<2 N /(N-2)$ as $n \rightarrow \infty$. This contradiction shows that $w=\left(w_{1}, w_{2}\right) \neq 0$.

On the other hand,

$$
\begin{aligned}
o(1)= & \int_{\Omega}\left[\nabla u_{n} \nabla \psi+\nabla v_{n} \nabla \varphi-\lambda \nabla u_{n} \nabla \varphi-\mu \nabla v_{n} \nabla \psi\right] d x \\
& -\int_{\Omega} f\left(x, u_{n}\right) \varphi d x-\int_{\Omega} g\left(x, v_{n}\right) \psi d x
\end{aligned}
$$

as $n \rightarrow \infty$ for $(\varphi, \psi) \in E$. Let

$$
p_{n}(x)=\left\{\begin{array}{ll}
\frac{f\left(x, u_{n}\right)}{u_{n}} & \text { if } u_{n}(x)>0 ; \\
0 & \text { if } u_{n}(x) \leq 0,
\end{array} \quad q_{n}(x)= \begin{cases}\frac{g\left(x, v_{n}\right)}{v_{n}} & \text { if } v_{n}(x)>0 \\
0 & \text { if } v_{n}(x) \leq 0\end{cases}\right.
$$

By $\left(H_{2}\right)-\left(H_{4}\right)$, we see that

$$
0 \leq p_{n}(x) \leq l, \quad 0 \leq q_{n}(x) \leq m, \quad \forall x \in \Omega,
$$

and there exist two functions $p(x), q(x) \in L^{\infty}(\Omega)$ such that

$$
p_{n} \rightarrow p, q_{n} \rightarrow q \text { in } L^{2}(\Omega)
$$

as $n \rightarrow \infty$. Hence

$$
p_{n}(x) w_{n}^{1} \rightarrow p(x) \max \left\{w^{1}(x), 0\right\}, q_{n}(x) w_{n}^{2} \rightarrow q(x) \max \left\{w^{2}(x), 0\right\} \text { in } L^{2}(\Omega)
$$

as $n \rightarrow \infty$. From (2.7), we have, for $(\varphi, \psi) \in E$, that

$$
\begin{aligned}
o(1)= & \int_{\Omega}\left[\nabla w_{n}^{1} \nabla \psi+\nabla w_{n}^{2} \nabla \varphi-\lambda \nabla w_{n}^{1} \nabla \varphi-\mu \nabla w_{n}^{2} \nabla \psi\right] d x \\
& -\int_{\Omega} p_{n}(x) w_{n}^{1} \varphi d x-\int_{\Omega} q_{n}(x) w_{n}^{2} \psi d x
\end{aligned}
$$


as $n \rightarrow \infty$. Letting $n \rightarrow \infty$, we obtain

$$
\begin{gathered}
\int_{\Omega}\left[\nabla w^{1} \nabla \psi+\nabla w^{2} \nabla \varphi-\lambda \nabla w^{1} \nabla \varphi-\mu \nabla w^{2} \nabla \psi\right] d x-\int_{\Omega} p(x) \max \left\{w^{1}, 0\right\} \varphi d x \\
-\int_{\Omega} q(x) \max \left\{w^{2}, 0\right\} \psi d x=0 .
\end{gathered}
$$

Therefore, $w^{1}, w^{2}$ satisfy

$$
\left\{\begin{array}{l}
-\Delta w^{1}+\mu \Delta w^{2}=q(x) \max \left\{w^{2}, 0\right\} \geq 0 \quad(x \in \Omega) \\
-\Delta w^{2}+\lambda \Delta w^{1}=p(x) \max \left\{w^{1}, 0\right\} \geq 0 \quad(x \in \Omega) .
\end{array}\right.
$$

Standard elliptic regularity theory in [4] shows that $w^{1}, w^{2} \in C^{2}(\Omega) \cap C(\bar{\Omega})$. By the strong maximum principle, we have $w^{1}-\mu w^{2}>0$ or $w^{1}-\mu w_{2}^{2}=0$ throught $\Omega$ and $w_{2}^{2}-w^{1}>0$ or $w^{2}-w^{1}=0$ throught $\Omega$. Since $w=\left(w_{1}, w_{2}\right) \neq 0$ and $0 \leq \lambda \mu<1$, we conclude that $w^{1}>0, w^{2}>0$ in $\Omega$. Hence $p(x)=l$, and $q(x)=m$. It follows that $w=\left(w^{1}, w^{2}\right)$ satisfies

$$
\int_{\Omega}\left[\nabla w^{1} \nabla \psi+\nabla w^{2} \nabla \varphi-\lambda \nabla w^{1} \nabla \varphi-\mu \nabla w^{2} \nabla \psi\right] d x-\int_{\Omega} l w^{1} \varphi d x-\int_{\Omega} m w^{2} \psi d x=0 .
$$

Let $k=\frac{m \lambda-\mu l+\sqrt{(m \lambda-\mu l)^{2}+4 m l}}{2 m}$. Observe that $w=\left(w^{1}, w^{2}\right)=\left(w^{1}, k \tilde{w}^{2}\right)$ satisfies

$$
\int_{\Omega}\left[\nabla w^{1} \nabla \psi-\mu k \nabla \tilde{w}^{2} \nabla \psi-m k \tilde{w}^{2} \psi\right] d x+k \int_{\Omega}\left[\nabla \tilde{w}^{2} \nabla \varphi-\frac{\lambda}{k} \nabla w^{1} \nabla \varphi-\frac{l}{k} w^{1} \varphi\right] d x=0 .
$$

Choosing $(\varphi, \psi)=\left(\frac{1}{k} \varphi_{1}, \varphi_{1}\right)$, we see that

$$
\int_{\Omega}\left[\nabla w^{1} \nabla \varphi_{1}-\mu k \nabla \tilde{w}^{2} \nabla \varphi_{1}-m k \tilde{w}^{2} \varphi_{1}\right] d x+\int_{\Omega}\left[\nabla \tilde{w}^{2} \nabla \varphi_{1}-\frac{\lambda}{k} \nabla w^{1} \nabla \varphi_{1}-\frac{l}{k} w^{1} \varphi_{1}\right] d x=0,
$$

that is,

$$
\left(1-\frac{\lambda}{k}\right) \int_{\Omega} \nabla\left(w^{1}+\frac{1-\mu k}{1-\frac{\lambda}{k}} \tilde{w}^{2}\right) \nabla \varphi_{1} d x=\frac{l}{k} \int_{\Omega}\left(w^{1}+\frac{m k^{2}}{l} \tilde{w}^{2}\right) \varphi_{1} d x .
$$

This contradicts the fact that $\lambda_{1}<\frac{\frac{l}{k}}{1-\frac{\lambda}{k}}=\frac{m \lambda+\mu l+\sqrt{(m \lambda-\mu l)^{2}+4 m l}}{2(1-\lambda \mu)}$. The assertion then follows.

Proposition 2.3. Suppose that $(H 1)-(H 5)$ hold and $\lambda_{1}<\frac{m \lambda+\mu l+\sqrt{(m \lambda-\mu l)^{2}+4 m l}}{2(1-\lambda \mu)}$, then problem (1.1) possesses at least one positive solution pair $(u, v) \in E$.

Proof. By Lemma 2.1, we know that the functional $I$ has an infinite dimensional linking geometry as described in Proposition 2.1. Proposition 2.1 implies that there exists a $(P S)_{c}$-sequence $\left\{z_{n}\right\}$ for $I$, where $c>0$. By Lemma 2.2, there exists a $z=(u, v)$ $\in E$ such that $z_{n} \rightarrow z=(u, v)$ in $E$ as $n \rightarrow \infty$. Now $z$ is a nontrivial solution of (1.1). The Strong Maximum Principle yields $u>0$ and $v>0$ in $\Omega$. The conclusion follows. 
By Proposition 2.3, we know that the set

$$
\{(u, v) \in E:(u, v) \not \equiv 0 \text { is a positive solution pair of }(1.1)\}
$$

is not empty. Let

$$
I^{\infty}=\inf \{I(u, v) \mid(u, v) \not \equiv 0 \text { is a positive solution pair of }(1.1)\} .
$$

Proposition 2.4. Suppose that $\lambda+\mu<2$. Then $I^{\infty}$ is attained and $I^{\infty}>0$.

Proof. By Proposition 2.3, we know that $I^{\infty}$ is finite. Indeed, by (2.4), Hölder inequality and the Sobolev embedding theorem, we get

$$
\begin{aligned}
\int_{\Omega}|\nabla u|^{2} d x & \leq \int_{\Omega}\left|g(x, v)\left\|u\left|d x+\mu \int_{\Omega}\right| \nabla u\right\| \nabla v\right| d x \\
& \leq \epsilon c\|u\|_{H_{0}^{1}}\|v\|_{H_{0}^{1}}+c_{\epsilon}\|u\|_{H_{0}^{1}}\|v\|_{L^{p}}^{p-1}+\mu\|u\|_{H_{0}^{1}}\|v\|_{H_{0}^{1}},
\end{aligned}
$$

Similarly,

$$
\int_{\Omega}|\nabla v|^{2} d x \leq \epsilon c\|u\|_{H_{0}^{1}}\|v\|_{H_{0}^{1}}+\tilde{c}_{\epsilon}\|v\|_{H_{0}^{1}}\|u\|_{L^{p}}^{p-1}+\lambda\|u\|_{H_{0}^{1}}\|v\|_{H_{0}^{1}} .
$$

Adding the two inequalities above we obtain

$$
\begin{aligned}
\int_{\Omega}\left(|\nabla u|^{2}+|\nabla v|^{2}\right) d x \leq & (\epsilon c+\lambda+\mu)\|u\|_{H_{0}^{1}}\|v\|_{H_{0}^{1}}+c_{\epsilon}\|u\|_{H_{0}^{1}}\|v\|_{L^{p}}^{p-1}+\tilde{c}_{\epsilon}\|v\|_{H_{0}^{1}}\|u\|_{L^{p}}^{p-1} \\
\leq & \frac{1}{2}(\epsilon c+\lambda+\mu) \int_{\Omega}\left(|\nabla u|^{2}+|\nabla v|^{2}\right) d x \\
& +c_{\epsilon}^{\prime}\left(\int_{\Omega}\left(|\nabla u|^{2}+|\nabla v|^{2}\right) d x\right)^{\frac{p}{2}}
\end{aligned}
$$

Since $\lambda+\mu<2$, it follows, by choosing $\epsilon>0$ suitably, that

$$
\int_{\Omega}\left(|\nabla u|^{2}+|\nabla v|^{2}\right) d x \geq c>0
$$

that is,

$$
\|z\| \geq c>0
$$

Suppose now that $z_{n}=\left(u_{n}, v_{n}\right) \not \equiv 0$ is a minimizing sequence of $I^{\infty}$. By Lemma 2.2 , we see that $\left\{z_{n}\right\}$ is uniformly bounded and relatively compact in $E$. Hence we may assume that $z_{n} \rightarrow z=(u, v)$ in $E$ and $I^{\prime}(z)=0$. (2.10) implies this $z \neq(0,0)$. It follows that $I^{\infty}=\lim _{n \rightarrow \infty} I\left(z_{n}\right)=I(z)>0$. Consequently, $I^{\infty}$ is attained by $z \in E \backslash\{0\}$. The proof is complete.

Proof of Theorem 1.1. This is a direct consequence of Proposition 2.3 and 2.4.

The proof of Theorem 1.2 and 1.3 are similar to that of Theorem 1.1. The main difference is to show that the $(P S)_{c}$ sequence is bounded. For instance, for Theorem 1.2, 
we may derive as (2.9) that

$$
\left\{\begin{array}{l}
-\Delta w^{1}-\mu \Delta w^{2}=q(x) \max \left\{w^{2}, 0\right\} \geq 0 \quad(x \in \Omega), \\
-\Delta w^{2}+\lambda \Delta w^{1}=p(x) \max \left\{w^{1}, 0\right\} \geq 0 \quad(x \in \Omega) .
\end{array}\right.
$$

Multiplying (2.11) by $w_{-}^{1}$ and integrating by parts, we obtain

$$
(1+\lambda \mu) \int_{\Omega}\left|w_{-}^{1}\right|^{2} d x \leq 0,
$$

implying that $w^{1} \geq 0$. Whence the strong maximum principle yields either (i) $w^{2} \equiv 0$ in $\Omega$ or (ii) $w^{2}>0$ in $\Omega$.

In the case (i), we have

$$
\Delta w^{1}=0
$$

this yields $w^{1} \equiv 0$ in $\Omega$, so that $\left(w^{1}, w^{2}\right)=0$, a contradiction.

In case (ii), $q(x)=m$ and $w^{1}, w^{2}$ satisfy

$$
-(1+\lambda \mu) \Delta w^{1}+\mu p(x) w^{1}=m w^{2} \geq 0,
$$

by the strong maximum principle. Also, $w^{1}>0$ or $\equiv 0$ in $\Omega$. If $w^{1} \equiv 0$ in $\Omega$, it follows that $\left(w^{1}, w^{2}\right)=0$, a contradiction to $\left(w^{1}, w^{2}\right) \neq 0$. If $w^{1}>0$ in $\Omega$, we have $p(x)=l$ and from (2.6) we see that $w=\left(w^{1}, w^{2}\right)$ satisfies

$$
\int_{\Omega}\left[\nabla w^{1} \nabla \psi+\nabla w^{2} \nabla \varphi-\lambda \nabla w^{1} \nabla \varphi+\mu \nabla w^{2} \nabla \psi\right] d x-\int_{\Omega} l w^{1} \varphi d x-\int_{\Omega} m w^{2} \psi d x=0 .
$$

The rest of the proof that the $(P S)_{c}$ sequence $\left\{z_{n}\right\}$ is bounded is similar to Lemma 2.2.

Finally, using Proposition 2.1, we see that problem (1.8) has a nontrivial nonnegative solution $(u, v)$ which satisfies

$$
-\Delta u-\mu \Delta v \geq 0, \quad-\Delta v+\lambda \Delta u \geq 0, \quad x \in \Omega .
$$

This implies that

$$
-(1+\lambda \mu) \Delta v \geq 0 .
$$

and so, by the maximum principle, either $v>0$ or $v=0$. The second case cannot happen since $v=0$ implies $u=0$. We remark that we could not show $u>0$ although we know $u \geq 0$. We only obtain $(u, v)$ is a nontrivial nonnegative solution.

ACKNOWLEDGEMENTS. The authors thank the referee for his/her valuable comments. This work was supported by NNSF of China, No: 10571175 and 10631030.

\section{REFERENCES}

1. Ph.Clement, D.G.Figueiredo and E.Mitidieri, Positive solutions of semilinear elliptic systems, Comm PDE., 17 (1992), 923-940.

2. D. G. Costa and O. H. Miyagaki, Nontrivial solutions for perturbations of the pLaplacian on unbounded dimains, J. Math. Anal. Appl. 193 (1995), 737-755. 
3. D. G. de Figueiredo and P. L. Felmer, On superquadratic elliptic systems, Trans. Amer. Math. Soc. 343 (1994), 99-106.

4. D. Gilbarg and N. S. Trudinger, Elliptic partial differential equations of second order. (Springer-Verlag, 1977).

5. J. Hulshof and R. C. A. M. Van de Vorst, Differential systems with strongly indefinite linear part, J. Functional Analysis 114 (1993), 32-58.

6. W. Kryszewski and A. Szulkin, Generalized linking theorem with an application to semilinear Schrödinger equations, Adv. Diff. Equas. 3 (1998), 441-472.

7. Gongbao Li and A. Szulkin, An asymptotically periodic Schrödinger equation with indefinite linear part. Comm. Contemp. Math. 4 (2002), 763-776.

8. Gongbao Li and Jianfu Yang, Asymptotically linear elliptic system. Comm PDE. 29 (2004), 925-954.

9. Gongbao $\mathrm{Li}$ and Huan-Song Zhou, The existence of a positive solution to asymptotically linear scalar field equations, Proc. Roy. Soc. Edinburgh Sect. A 130 (2000), 81-105.

10. Chaoquan Peng and Jianfu Yang, Nonnegative solutions for nonlinear elliptic systems, J. Math. Anal. Appl. to appear.

11. M. Willem, Minimax theorems (Birkhauser, 1996).

12. Huan-Song Zhou, An application of mountain pass theorem, Acta Math. Sinica, (N. S), 18 (2002), 27-36.

13. R. C. A. M. Van de Vorst, Variational identities and applications to differential systems, Arch. Rational Mech. Anal. 116 (1991), 375-398. 